\title{
Hybrid Conducting Polymer-Hydrogel Conduits for Axonal Growth and Neural Tissue Engineering
}

\author{
Mohammad R. Abidian,* Eugene D. Daneshvar, Brent M. Egeland, Daryl R. Kipke, \\ Paul S. Cederna, and Melanie G. Urbanchek
}

Peripheral nerve lesions are estimated to be $2.8 \%$ of all trauma cases and may result in permanent loss of motor and sensory function. ${ }^{[1]}$ In the United States, approximately 360000 people suffer from peripheral nerve trauma. ${ }^{[2]}$ Most severe injuries are associated with nerve gaps, which prevent regenerating axons from effectively innervating the distal motor and sensory end organs. Autografts are the clinical "gold standard" for bridging peripheral nerve gaps and have been widely used experimentally and clinically for reconstruction..$^{[3]}$ The disadvantages of this technique include a limited supply of donor nerve making it impossible to reconstruct complex nerve gaps, sensory deficits in the distribution of the donor nerve, painful dysesthesias following sensory nerve harvest, and structural/ultrastructural nerve mismatch. ${ }^{[4]}$

To overcome the limitations associated with peripheral nerve autografts, artificial conduits that provide a suitable environment for axonal regeneration have been developed. ${ }^{[5]}$ Artificial conduits have been constructed and clinically used for repairing peripheral nerve gaps ${ }^{[6]}$ from biodegradable materials ${ }^{[7]}$ and nonbiodegradable materials. ${ }^{[8]}$ Nerve conduits must fulfill several requirements: i) they should be biocompatible, ii) have sufficient mechanical stability during nerve regeneration, and iii) allow transport of nutrients and waste product. ${ }^{[4,5,9,10]}$ Hydrogels are promising candidate materials for nerve conduits ${ }^{[11-16]}$ because these materials are biocompatible, permeable, and exhibit physical characteristics similar to those of soft tissue. ${ }^{[17,18]}$ Agarose hydrogel, a natural biocompatible polysaccharide, has been considered for fabrication of nerve conduits. ${ }^{[19]}$ In vitro studies have shown that agarose promotes adhesion, survival, and

Prof. M. R. Abidian

Department of Bioengineering

Materials Science \& Engineering

and Chemical Engineering

Pennsylvania State University

University Park, PA 16802, USA

E-mail: mabidian@psu.edu

E. D. Daneshvar, Prof. D. R. Kipke

Department of Biomedical Engineering

University of Michigan

Ann Arbor, MI 48109, USA

Dr. B. M. Egeland, Prof. P. S. Cederna, Prof. M. G. Urbanchek

Section of Plastic Surgery

Department of Surgery

University of Michigan

Ann Arbor, MI 48109, USA neurite outgrowth of nerve cells. ${ }^{[20]}$ Moreover, agarose nerve conduits support axon elongation and functional improvement during nerve regeneration in a $2 \mathrm{~mm}$ spinal cord lesion in rats. ${ }^{[21]}$ However, a drawback of hydrogel conduits for longer nerve gaps is their lack of mechanical integrity (strength) under physiological conditions. ${ }^{[18,22]}$ Swelling of hydrogels has been shown in both in vitro and in vivo studies to close the conduit cavities and block axonal growth over a long-term study (12 weeks) and larger gap sizes $(10 \mathrm{~mm}) \cdot{ }^{[11,23,24]}$ Belkas et al. reported that synthetic hydrogel poly(2-hydroxyethyl methacrylate-co-methyl methacrylate) (PHEMA-MMA) tubes implanted in $10 \mathrm{~mm}$ rat sciatic nerve gaps after 16 weeks collapsed and prevented successful axonal regeneration and elongation. Four of ten reconstructed nerve gaps lacked a regenerating cable at 16 weeks likely because the PHEMA-MMA conduits collapsed, physically obstructing axonal regeneration. ${ }^{[11]}$ To overcome this issue, Katayama et al. designed PHEMA-MMA coil-reinforced composite tubes and demonstrated a significant improvement in the long term performance of modified tubes in a rat sciatic nerve gap model. ${ }^{[25]}$

Conducting polymers such as poly(pyrrole) (PPy) and poly(3,4-ethylenedioxythiophene) (PEDOT) have been considered for biomedical applications, ${ }^{[26,27]}$ in particular, neural interfaces ${ }^{[28-33]}$ due to the following four characteristics: 1) their organic nature, ${ }^{[26]} 2$ ) their response to electrical stimuli (volume, color, and wettability changes), ${ }^{[34]} 3$ ) their ability to be functionalized with biomolecules, ${ }^{[35]}$ and 4) their ionic and electronic conductivity. ${ }^{[26,36,37]}$ PEDOT has an excellent chemical stability in aqueous solution at room temperature even at higher temperature, which originates from the stabilizing effect on the positive charges by sulfur and oxygen. ${ }^{[38]}$ Due to the lack of biodegradability of conducting polymers, development of methods to introduce biodegradability to conducting polymers is a very important and challenging task for the fabrication of tissue engineering scaffolds. These methods include addition of hydrolyzable side groups to the monomer ${ }^{[39]}$ and creation of conducting polymer-biodegradable polymer composites. ${ }^{[40-42]}$ In vitro studies have demonstrated that PPy and PEDOT can promote neurite outgrowth by providing physical and/or electrical growth cues. ${ }^{[43-47]}$ To evaluate the biocompatibility of PPy and PEDOT with neural tissue, recently we cultured primary dorsal root ganglion (DRG) explants on PPy and PEDOT films and their nanotube counterparts. ${ }^{[48]}$ DRG explants successfully attached and grew neurites on all conducting polymer substrates. All substrates supported neurite outgrowth in a radial direction away from the ganglia, however, neurites appeared qualitatively longer, thinner, and less branched on a PEDOT

DOI: 10.1002/adhm.201200182 
film and nanotubes. Various attempts have been made to develop conductive conduits using PPy and biodegradable polymers. Some examples include PPy-chitosanpolycaprolactone membrane ${ }^{[49]}$ and $\mathrm{PPy}-$ polycaprolactone fumarate composite conduits. ${ }^{[41]}$ PPy conduits have also been used for in vivo studies. ${ }^{[50,51]}$ For example Zheng et al. fabricated PPy-poly(D,L-lactide-co-caprolactone) conduits and implanted them in $8 \mathrm{~mm}$ sciatic nerve gaps. Their animal experiments demonstrated that the sciatic nerves were regenerated in the PPy composite conduits and the regenerated nerve tissue displayed a structure typical of normal sciatic nerves with myelinated and unmyelinate axons and Schwann cells. ${ }^{[51]}$

Here we report for the first time an application of PEDOT traces within hydrogel nerve conduits for axonal regeneration. This study describes a novel method for the preparation of mechanically reinforced agarose nerve conduits that are further made conductive by use of a thin layer of PEDOT. We hypothesize that PEDOT-modified agarose conduits support superior neural regeneration as compared to the plain agarose conduits. PEDOT has emerged as the most promising conducting polymer for neural interfaces since it exhibits a very high conductivity and stability in the oxidized state. ${ }^{[38,52]}$ To investigate the influence of PEDOT on the axonal regeneration across nerve tubes we developed an innovative hybrid conduit consisting of electrically polymerized PEDOT and agarose hydrogel. PEDOT was electrodeposited inside the lumen to create a fully coated-PEDOT agarose (FPEDOTA) conduit and a partially coated-PEDOT agarose (PPEDOTA) conduit. PEDOT polymerization within a hydrogel has been shown to mechanically strengthen the combination. ${ }^{[53]}$ Three animal groups (five rats each) were compared with autograft controls: animals that received plain agarose (PA), FPEDOTA, and PPEDOTA conduits. Experimental conduits were implanted in $10 \mathrm{~mm}$ peroneal nerve gaps in rats using mechanically supportive stents and were evaluated 12 weeks postoperatively. The outcome measures utilized included extensor digitorum longus (EDL) muscle contractile force measurements, a muscle innervated by the peroneal nerve, and nerve histomorphometry. We demonstrated that the hybrid conducting polymer-hydrogel conduits (FPEDOTA and PPEDOTA) supported superior neural regeneration as compared to the plain hydrogel conduits (PA).

In this study, we used a soft thermosensitive agarose hydrogel because it is optically transparent and permeable to the surrounding tissue medium, and has been used as a spinal cord surrogate. ${ }^{[5]}$ General schemes for producing FPEDOTA and PPEDOTA conduits are illustrated in Figure 1. Briefly, the fabrication process begins with $800 \mu \mathrm{m}$ diameter polystyrene (PS) template fibers (Figure 1A, H). PS fibers were then sputter coated with a very thin layer $(\sim 100 \AA)$ of gold-palladium (AuPd) (Figure 1B). In the case of PPEDOTA, strips of $1 \mathrm{~mm}$ width masking tape were wrapped in a spiral fashion around the PS fibers (Figure 1I), they were then sputter coated with AuPd (Figure 1J) and the masking tape was removed (Figure $1 \mathrm{~K}$ ). Agarose hydrogel is a soft and permeable material but is not flexible and may break apart when suturing. Therefore two stainless steel stents were placed at both the proximal and distal ends of the PS fiber to provide suturing support of the conduit (see Supporting Information, Figure S1). The AuPd-coated PS template fibers were then dipped into the agarose solution (Figure 1C, L). The agarose gelled quickly at room temperature to create a thin layer of hydrogel (Figure 1D, M). This dipping process was repeated until the conduit achieved adequate thickness. The thickness of the agarose was controlled in the range of $0.85 \pm 0.27 \mathrm{~mm}$. The length of the conduit was controlled by how much of the template fiber was submerged in the agarose solution.

Once the hydrogel was formed on the coated template fiber, PEDOT was grown on the surface of the AuPd-coated PS fibers by an electrochemical deposition process at a current density of $0.5 \mathrm{~mA} \mathrm{~cm}^{-2}$ for $30 \mathrm{~min}$ (Figure 1E, N). In this stage, the thickness of the PEDOT layer was measured at $6 \pm$ $1 \mu \mathrm{m}$ for FPEDOTA and $10 \pm 2 \mu \mathrm{m}$ for PPEDOTA (deposition charge density $900 \mathrm{mC} \mathrm{cm}^{-2}$ ). We initially electrodeposited PEDOT on the surface of AuPd before removing the PS fibers (Figure 1E, N). Since the agarose hydrogel is porous and permeable, EDOT monomers were available for polymerization at the metal surface of the template fiber and through the hydrogel. ${ }^{[29,55]}$ Dissolving the PS fibers before PEDOT 
deposition would result in cracking in the thin AuPd film. We found that the initial electrodeposition of PEDOT on AuPd provided physical support for the thin layer of $\mathrm{AuPd}$ and remained intact after the subsequent dissolving process of the PS core fibers. This layer also resulted in a conductive pathway through the length of the tube. The PS template fibers were dissolved to the length of the hydrogel tube by soaking in a tetrahydrofuran bath overnight (Figure 1F, O). Dissolving the template fiber opened the lumen of the tube for electrodeposition of PEDOT on the surface of AuPd within the inner lumen with the same deposition charge density (Figure 1G, P). Figure 1Q, R and Figure $1 \mathrm{~S}, \mathrm{~T}$ are optical micrographs of FPEDOTA and PPEDOTA conduits, respectively. Red arrows shown in Figure 1R indicate the cloudy-like growth of PEDOT within the agarose scaffold. We have previously demonstrated that PEDOT could be grown through the alginate-coated neural electrodes. [29] The total thickness of the PEDOT layer was measured $11 \pm 2 \mu \mathrm{m}$ for FPEDOTA and $18 \pm$ $2 \mu \mathrm{m}$ for PPEDOTA (total charge deposition of $1800 \mathrm{mC} \mathrm{cm}^{-2}$ ). All of the peripheral nerve conduits were prepared according to the above fabrication process. The total length of the conduits was selected as $12 \mathrm{~mm}, 10 \mathrm{~mm}$ gap length, with $1 \mathrm{~mm}$ of overlap at the proximal and distal coaptation site.

The nerve guidance channels were implanted in $10 \mathrm{~mm}$ peroneal nerve gaps in a total 15 rats (each 5 rats) (Figure 2A-D) and evaluated 12 weeks postoperatively. In Figure $2 \mathrm{E}$ and Figure $2 \mathrm{~F}$, we compared the functional recovery of the nerve gap conduits using EDL muscle mass and EDL maximal specific muscle force (maximal muscle force normalized to muscle mass) measurements. EDL muscles reinnervated by peroneal nerve autografts had the maximal muscle mass (107 $\pm 30 \mathrm{mg}$ ). There was a decline in reinnervated EDL muscle mass when reinnervated by one of the synthetic conduits, however, EDL muscle mass was $60 \pm 11$ and $54 \pm 6 \mathrm{mg}$ for PPEDOTA and FPEDOTA, respectively; significantly higher than PA $(26 \pm$ $4 \mathrm{mg}, P<0.05$ ) (Figure 2E). Under optimal conditions, using a peripheral nerve autograft, the reinnervated EDL muscle generated a specific force of $19.7 \pm 7.2 \mathrm{~N} \mathrm{~g}^{-1}$ of maximum tetanic isometric force. There was a substantial decline in the EDL maximal specific muscle force when the nerve gaps were reconstructed with either FPEDOTA or PPEDOTA $(2.4 \pm$ 3.3 and $4.2 \pm 9.2 \mathrm{~N} \mathrm{~g}^{-1}$, respectively), however, there was still evidence of end organ reinnervation and force generation, demonstrating the utility of this hybrid conduit for functional reconstruction of nerve gaps. Interestingly, there was no functional reinnervation of the EDL when nerve gaps were reconstructed with PA conduits (Figure 2F).
Evaluation of the axonal sprouting and elongation within the nerve conduits following nerve gap reconstruction demonstrated outstanding neural regeneration in the nerve autograft group with large numbers of myelinated axons, a high percentage of neural tissue, and large diameter axons (Figure 3A, E). No nerve fibers were seen at midgraft in the PA group (Figure 3B, F), which was consistent with the low EDL specific muscle force measurements in this group. In the FPEDOTA and PPEDOTA groups, the axonal regeneration appeared robust with mature, large diameter, myelinated axons. Regeneration in the PPEDOTA group (Figure 3D, H) appeared slightly more extensive than the FPEDOTA group (Figure 3C, G). As shown in Table 1, the ratio of the axoplasm to nerve fiber area indicates that nerve fibers were growing through the synthetic grafts with normal proportions of axoplasm and myelin (Schwann cell size) for all but the PA group. Nerve fiber diameter, axoplasm diameter, and myelin thickness measurements indicate that the FPEDOTA groups have significantly $(p \leq 0.05)$ smaller nerve 

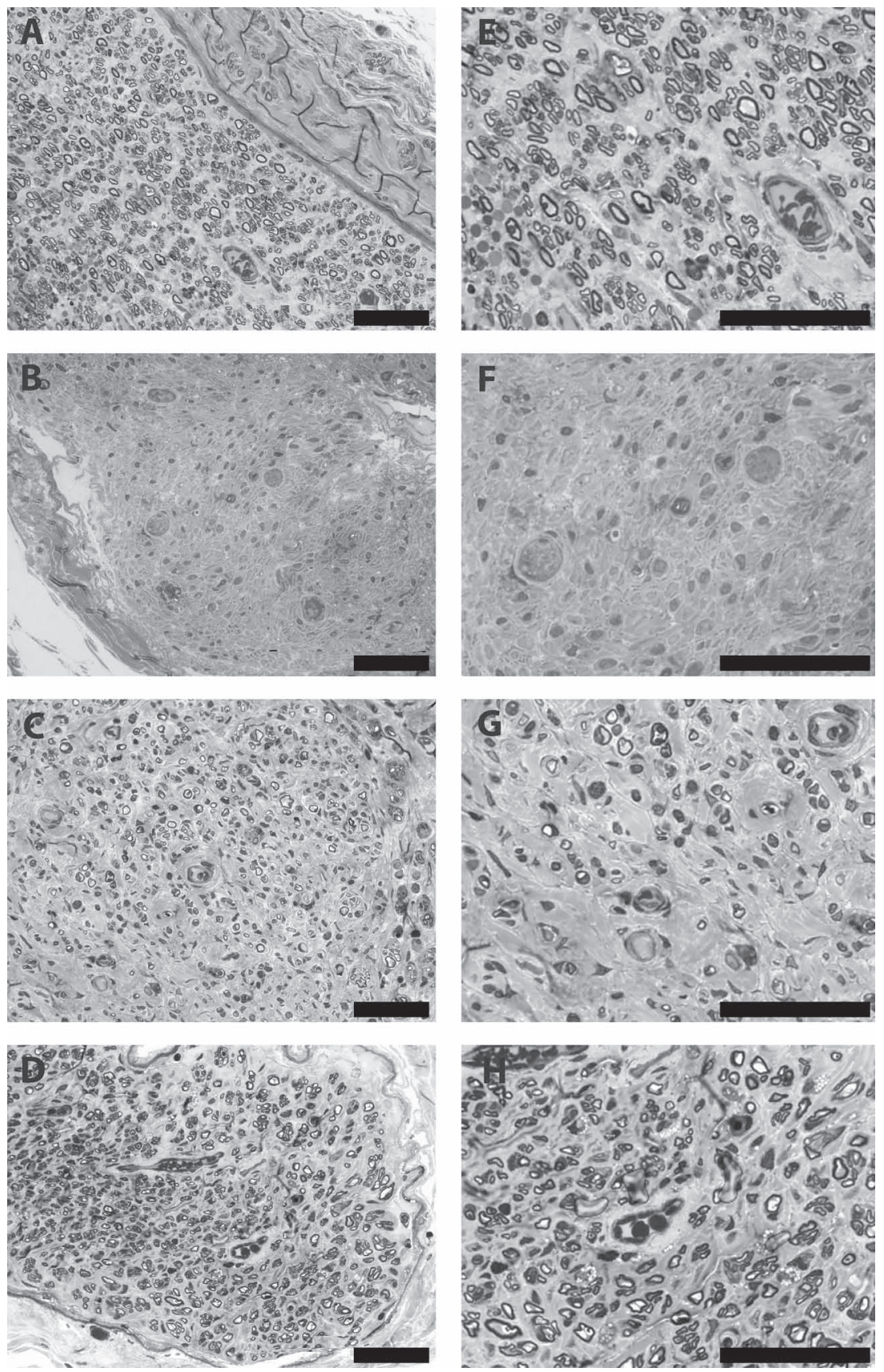

Figure 3. Optical micrographs of peripheral nerves at midgraft. A, E) Autograft. B, F) PA. C, G) FPEDOTA. D, H) PPEDOTA. Scale bars are $50 \mu \mathrm{m}$.

fibers, less axoplasm, and less myelin around nerve fibers. On the other hand, the PPEDOTA group nerve fibers displayed a similar morphology to the autograft group (Table 1).

Although the type of hydrogel we used as a conduit material for peripheral nerve regeneration is different from those previously reported by others, ${ }^{[56,57]}$ we noted no axonal regeneration in the PA group, consistent with the findings of Shoichet's group. ${ }^{[3,11]}$ In fact, similarly we observed that the PA conduits had collapsed, physically blocking axonal regeneration due to the swelling of the agarose hydrogel. Therefore, we electrodeposited two layers of PEDOT (Figure 1E and $\mathrm{G}$ for FPEDOTA and Figure $1 \mathrm{~N}$ and $\mathrm{P}$ for PPEDOTA), to enhance the conduit strength and rigidity to maintain the integrity of the conduit lumen, thus avoiding physical obstruction of axonal elongation. It has been demonstrated that PEDOT growth within the hydrogel could significantly improve the mechanical property of the hydrogel. ${ }^{[53]}$ This can also explain the superior performance of our conductive hydrogels.

In our study we did not observe PEDOT degradation and delamination from the thin AuPd layer. Histology results confirmed that PEDOT films remained intact and adherent after 12 weeks of implantation, consistent with that previously reported for in vivo assessments ${ }^{[50]}$ and under simulated physiological conditions in vitro. ${ }^{[58]}$ In combination, the EDL muscle force measurements and histomorphometric analysis of axonal regeneration demonstrated that the novel hybrid conducting polymer-hydrogel conduits support neural regeneration across $10 \mathrm{~mm}$ peroneal nerve gaps. Although we did not observe any negative effect on the regenerated nerves by using non-degradable stents, we suggest biodegradable stents for the design of degradable conductive-hydrogel conduits for longterm nerve regeneration.

The novel nerve construct presented here demonstrates for the first time the use of hybrid conducting polymer-hydrogel conduits (PEDOT-agarose) for axonal regeneration. In this study, we investigated the influence of a PEDOT coating layer on the axonal regeneration in a $10 \mathrm{~mm}$ nerve gap in rats. Although both FPEDOTA and PPEDOTA conduits supported axonal growth, PPEDOTA demonstrated slightly better performance presumably due to better diffusion of nutrients and biomolecules into and within the lumen of the conduits. In longer peripheral nerve gaps, axonal regeneration is limited by the lack of neurotrophic support and appropriate adhesion molecules, which are essential for migration of Schwann cells into the nerve gap to support axon elongation and myelination. ${ }^{[59,60]}$ Several strategies have been developed to stimulate Schwann cell migration and axonal growth. These strategies focus on utilizing topographical cues such as extracellular matrix (ECM) protein and biodegradable fibers, ${ }^{[61,62]}$ biochemical factors such as growth factors and ECM molecules, ${ }^{[63,64]}$ electrical stimulation, ${ }^{[65,66]}$ and cell transplantations such as Schwann cells or stem cells. ${ }^{[67,68]}$ Since previous studies have demonstrated the benefits of conducting polymers for in vitro neurite outgrowth by providing electrical, physical, and biochemical cues, ${ }^{[43,45-47,69,70]}$ this study paves the way for the design of a three-dimensional conductive hydrogel scaffold 
Table 1. Summary data for nerve conduit cross section morphology at midgraft.

\begin{tabular}{lcccc}
\hline \multirow{2}{*}{$\begin{array}{l}\text { Dependent Nerve } \\
\text { Variables }\end{array}$} & \multicolumn{4}{c}{ Type of Conduit ${ }^{\mathrm{a})}$} \\
\cline { 2 - 5 } & Autograft & PA $^{\mathrm{b})}$ & PPEDOTA & FPEDOTA \\
\hline Axoplasm/nerve fiber area & $0.53 \pm 0.09$ & - & $0.41 \pm 0.02$ & $0.45 \pm 0.05$ \\
Nerve fiber diameter $[\mu \mathrm{m}]$ & $8.85 \pm 3.09$ & - & $4.01 \pm 0.31$ & $3.34 \pm 0.61^{\mathrm{c})}$ \\
Axoplasm diameter $[\mu \mathrm{m}]$ & $4.72 \pm 2.02$ & - & $1.64 \pm 0.06$ & $1.53 \pm 0.41^{\mathrm{c})}$ \\
Myelin thickness $[\mu \mathrm{m}]$ & $2.06 \pm 0.74$ & - & $1.18 \pm 0.12$ & $\left.0.91 \pm 0.13^{\mathrm{c}}\right)$ \\
\hline
\end{tabular}

a) Mean \pm standard deviation. GLM statistic with Tukey multiple comparison correction when main effect was significant. Significance set a priori at $0.10 ;{ }^{b}$ No nerve fibers were seen at midgraft in the PA group; ${ }^{c}$ Different from autograft group.

for accelerated, directional, and controlled axonal growth in the peripheral nervous systems. PEDOT is not degradable, and materials that remain in the body long-term may induce chronic inflammation and require surgical removal. With development of biodegradable conducting polymers ${ }^{[71]}$ and application of biodegradable hydrogels and stents, the hybrid conduit presented here can ultimately be used for neural tissue regeneration. Another potential application of this hybrid conductive construct is in designing a more biocompatible and seamless biotic-abiotic peripheral nerve interface for motor control and sensory feedback from advanced neural prosthetic devices. The ability of mixed ionic-electronic conduction of conducting polymers can facilitate the signal transduction between the ionically conductive biologic tissues and the electronically conductive electrodes. The PEDOT lining may be used to facilitate future studies using electrical stimulation and/or controlled release of neurotrophins. In addition to promoting axonal growth, the conductive lining may be used as an effective interface between the electronic circuitry of neural prosthetic devices and the peripheral nervous system.

\section{Experimental Section}

Materials: 3,4-Ethylenedioxythiophene (EDOT, BAYTRON $\mathbb{R}$ M) with molecular weight $142.17 \mathrm{~g} \mathrm{~mol}^{-1}$ was received from H.C. Starck Inc. (Newton, MA). Lithium perchlorate $\left(\mathrm{LiClO}_{4}\right)$, tetrahydrofuran (THF), and agarose type VII were purchased from Sigma-Aldrich. Polystyrene (PS) fibers with a diameter of $800 \mu \mathrm{m}$ were prepared by Paradigm Optics (Vancouver, WA). Stainless steel surgical stents were purchased from Pulse Systems Inc. (Concord, CA).

Fabrication of Fully Coated PEDOT Agarose Conduit: The templating process began with $800 \mu \mathrm{m}$ diameter PS fibers. PS fibers were cut to a length of approximately $3-4 \mathrm{~cm}$ and were then sputter coated with AuPd. The body of the tube was created using an agarose hydrogel. Powered agarose $(1.9 \mathrm{~g})$ and $40 \mathrm{~mL}$ of deionized water was heated until boiling, while mixing to ensure all agarose had been dissolved to create a $4.5 \%$ hydrogel solution. This solution was degassed under vacuum before it was used further. The coated template fibers were then dipped into the agarose solution and removed. The agarose gelled quickly at room temperature to create a thin layer of hydrogel. This dipping process was repeated until the tube achieved adequate thickness and length. The length of the tube is controlled by how much of the template fiber was submerged in the agarose solution. Once the hydrogel was formed on the coated template fiber, a conducting polymer could be grown on the inner surface of the tube. The electrochemical deposition was performed by an Autolab PGSTAT-12 (EcoChemie, Utrecht, Netherlands) in galvanostatic mode with a conventional two electrode configuration at room temperature. Conducting polymer deposition was carried out in a $0.01 \mathrm{~m}$ EDOT and $0.1 \mathrm{M} \mathrm{LiClO}_{4}$ aqueous solution at a current density of $0.5 \mathrm{~mA} \mathrm{~cm}^{-2}$ for $30 \mathrm{~min}$. The amount of polymer coated on the inner tube was controlled by the total charge passed during polymerization. The working and sensing electrodes were connected to the AuPd-coated PS fiber while the reference and counter electrodes were connected to a platinum wire within the $\mathrm{EDOT} / \mathrm{LiClO}_{4}$ solution. After electropolymerization of the PEDOT, the template fiber was trimmed to the length of the hydrogel tube. The tube was placed in a THF bath overnight in order to dissolve the template fiber. Dissolving the template fiber opened the lumen of the tube.

Animal Model, Measurement of Contractile Properties, Nerve Histology, and Statistical Analysis are given in the Supporting Information. All animal procedures were approved by The University of Michigan's University Committee on Use and Care of Animals and were in accordance with the National Institute of Health guidelines.

\section{Supporting Information}

Supporting Information is available from the Wiley Online Library or from the author.

\section{Acknowledgements}

The authors thank David Turer, David Thompson, Rachel Miriani, and Melissa Wright for their assistance with fabrication of conduits. The authors thank Yamini Jadcherla, Katie Ewing, and Kirsten Schroeder for their assistance with nerve morphometry, and physiological outcomes. The authors acknowledge Army Research Office MURI (Contract Number W911NF-06-1-0218, Proposal Number 50376-LS-MUR) and Pennsylvania State University start up fund. The authors have invention disclosures and patent applications related to these materials pending with The University of Michigan Office of Technology Transfer and the U. S. Patent and Trademark Office.

Received: May 26, 2012

Revised: June 26, 2012

Published online: August 27, 2012

[1] J. Noble, C. A. Munro, V. Prasad, R. Midha, J. Trauma 1998, 45, 116.

[2] J. Kelsey, A. Praemer, L. Nelson, A. Felberg, D. Rice, Upper Extremity Disorders: Frequency, Impact, and Cost, Churchill-Livingstone, London 1997.

[3] J. S. Belkas, M. S. Shoichett, R. Midha, Neurol. Res. 2004, 26, 151.

[4] T. Matsuyama, M. Mackay, R. Midha, Neurol. Med.-Chirurgica 2000, 40, 187.

[5] V. B. Doolabh, M. C. Hertl, S. E. Mackinnon, Rev. Neurosci. 1996, 7, 47.

[6] M. F. Meek, J. H. Coert, J. Reconstr. Microsurg. 2002, 18, 97.

[7] G. R. D. Evans, K. Brandt, S. Katz, P. Chauvin, L. Otto, M. Bogle, B. Wang, R. K. Meszlenyi, L. C. Lu, A. G. Mikos, C. W. Patrick, Biomaterials 2002, 23, 841 .

[8] Q. Zhao, G. Lundborg, N. Danielsen, L. M. Bjursten, L. B. Dahlin, Brain Res. 1997, 769, 125.

[9] F. J. Rodriguez, N. Gomez, G. Perego, X. Navarro, Biomaterials 1999, 20, 1489.

[10] C. E. Schmidt, J. B. Leach, Annu. Rev. Biomed. Eng. 2003, 5, 293.

[11] J. S. Belkas, C. A. Munro, M. S. Shoichet, R. Midha, Restor. Neurol. Neuros. 2005, 23, 19.

[12] P. D. Dalton, L. Flynn, M. S. Shoichet, Biomaterials 2002, 23, 3843. 
[13] L. A. Pfister, M. Papaloizos, H. P. Merkle, B. Gander, J. Biomed. Mater. Res. A 2007, 80A, 932.

[14] S. Stokols, J. Sakamoto, C. Breckon, T. Holt, J. Weiss, M. H. Tuszynski, Tissue Eng. 2006, 12, 2777.

[15] E. C. Tsai, P. D. Dalton, M. S. Shoichet, C. H. Tator, Biomaterials 2006, 27, 519.

[16] T. T. Yu, M. S. Shoichet, Biomaterials 2005, 26, 1507.

[17] J. L. Drury, D. J. Mooney, Biomaterials 2003, 24, 4337.

[18] K. Y. Lee, D. J. Mooney, Chem. Rev. 2001, 101, 1869.

[19] G. Perale, F. Rossi, E. Sundstrom, S. Bacchiega, M. Masi, G. Forloni, P. Veglianese, ACS Chem. Neurosc. 2011, 2, 336.

[20] Y. Luo, M. S. Shoichet, Nat. Mater. 2004, 3, 249.

[21] S. Stokols, M. H. Tuszynski, Biomaterials 2006, 27, 443.

[22] B. V. Slaughter, S. S. Khurshid, O. Z. Fisher, A. Khademhosseini, N. A. Peppas, Adv. Mater. 2009, 21, 3307.

[23] G. C. de Ruiter, I. A. Onyeneho, E. T. Liang, M. J. Moore, A. M. Knight, M. J. A. Malessy, R. J. Spinner, L. Lu, B. L. Currier, M. J. Yaszemski, A. J. Windebank, J. Biomed. Mater. Res. A 2008, 84A, 643.

[24] G. C. de Ruiter, R. J. Spinner, M. J. A. Malessy, M. J. Moore, E. J. Sorenson, B. L. Currier, M. J. Yaszemski, A. J. Windebank, Neurosurgery 2008, 63, 144 .

[25] Y. Katayama, R. Montenegro, T. Freier, R. Midha, J. S. Belkas, M. S. Shoichet, Biomaterials 2006, 27, 505.

[26] M. Berggren, A. Richter-Dahlfors, Adv. Mater. 2007, 19, 3201.

[27] N. K. Guimard, N. Gomez, C. E. Schmidt, Prog. Polym. Sci. 2007, 32, 876.

[28] R. A. Green, N. H. Lovell, G. G. Wallace, L. A. Poole-Warren, Bioma terials 2008, 29, 3393

[29] M. R. Abidian, D. C. Martin, Adv. Funct. Mater. 2009, 19, 573.

[30] M. R. Abidian, K. A. Ludwig, T. C. Marzullo, D. C. Martin, D. R. Kipke, Adv. Mater. 2009, 21, 3764.

[31] M. R. Abidian, D. H. Kim, D. C. Martin, Adv. Mater. 2006, 18, 405.

[32] K. A. Ludwig, J. D. Uram, J. Yang, D. C. Martin, D. R. Kipke, J. Neural Eng. 2006, 3, 59.

[33] M. G. Urbanchek, B. Wei, B. M. Egeland, M. R. Abidian, D. R. Kipke, P. S. Cederna, Plast. Reconstr. Surg. 2011, 128, 270E.

[34] W. Lu, A. G. Fadeev, B. H. Qi, E. Smela, B. R. Mattes, J. Ding, G. M. Spinks, J. Mazurkiewicz, D. Z. Zhou, G. G. Wallace, D. R. MacFarlane, S. A. Forsyth, M. Forsyth, Science 2002, 297 983.

[35] P. Tengvall, I. Lundstrom, B. Liedberg, Biomaterials 1998, 19, 407.

[36] C. Deslouis, T. ElMoustafid, M. M. Musiani, B. Tribollet, Electrochim. Acta 1996, 41, 1343.

[37] X. M. Ren, P. G. Pickup, J. Chem. Soc., Faraday Trans. 1993, 89, 321.

[38] G. Heywang, F. Jonas, Adv. Mater. 1992, 4, 116.

[39] A. N. Zelikin, D. M. Lynn, J. Farhadi, I. Martin, V. Shastri, R. Langer, Angew. Chem. Int. Ed. 2002, 41, 141

[40] L. H. Huang, J. Hu, L. Lang, X. Wang, P. B. Zhang, X. B. Jing, X. H. Wang, X. S. Chen, P. I. Lelkes, A. G. MacDiarmid, Y. Wei, Biomaterials 2007, 28, 1741

[41] M. B. Runge, M. Dadsetan, J. Baltrusaitis, A. M. Knight, T. Ruesink, E. A. Lazcano, L. C. Lu, A. J. Windebank, M. J. Yaszemski, Biomaterials 2010, 31, 5916

[42] Z. X. Wang, C. Roberge, Y. Wan, L. H. Dao, R. Guidoin, Z. Zhang, J. Biomed. Mater. Res. A 2003, 66A, 738.

[43] A. F. Quigley, J. M. Razal, B. C. Thompson, S. E. Moulton, M. Kita, E. L. Kennedy, G. M. Clark, G. G. Wallace, R. M. I. Kapsa, Adv. Mater. 2009, 21, 4393
[44] T. Nyberg, O. Inganas, H. Jerregard, Biomed. Microdevices 2002, 4, 43.

[45] M. B. Runge, M. Dadsetan, J. Baltrusaitis, T. Ruesink, L. C. Lu, A. J. Windebank, M. J. Yaszemski, Biomacromolecules 2010, 11 2845.

[46] J. W. Xie, M. R. MacEwan, S. M. Willerth, X. R. Li, D. W. Moran, S. E. Sakiyama-Elbert, Y. N. Xia, Adv. Funct. Mater. 2009, 19, 2312

[47] C. E. Schmidt, V. R. Shastri, J. P. Vacanti, R. Langer, Proc. Natl. Acad. Sci. USA 1997, 94, 8948

[48] M. R. Abidian, J. M. Corey, D. R. Kipke, D. C. Martin, Small 2010, 6, 421.

[49] Y. Wan, J. A. Gao, J. Zhang, W. M. Peng, G. F. Qiu, Polym. Degrad. Stab. 2010, 95, 1994.

[50] P. M. George, R. Saigal, M. W. Lawlor, M. J. Moore, D. A. LaVan, R. P. Marini, M. Selig, M. Makhni, J. A. Burdick, R. Langer, D. S. Kohane, J. Biomed. Mater. Res. A 2009, 91A, 519.

[51] Z. Zhang, M. Rouabhia, Z. Wang, C. Roberge, G. Shi, P. Roche, J. Li, L. H. Dao, Artif. Organs 2007, 31, 13.

[52] B. L. Groenendaal, F. Jonas, D. Freitag, H. Pielartzik, J. R. Reynolds, Adv. Mater. 2000, 12, 481.

[53] R. A. Green, R. T. Hassarati, J. A. Goding, S. Baek, N. H. Lovell, P. J. Martens, L. A. Poole-Warren, Macromol. Biosci. 2012, 12, 494.

[54] R. Bellamkonda, J. P. Ranieri, P. Aebischer, J. Neurosci. Res. 1995, 41, 501.

[55] D. R. Kim, M. Abidian, D. C. Martin, in Architecture and Application of Biomaterials and Biomolecular Materials, Vol. 1 (Eds: J. Y. Wong, A. L. Plant, C. E. Schmidt, L. Shea, A. J. Coury, C. S. Chen, A. E. Barron, H. A. Klok, W. M. Saltzman, A. Chilkoti, D. Luo, K. Uhrich), 2004, 55.

[56] Y. Luo, P. D. Dalton, M. S. Shoichet, Chem. Mater. 2001, 13, 4087.

[57] E. C. Tsai, P. D. Dalton, M. S. Shoichet, C. H. Tator, J. Neurotrauma 2004, 21, 789

[58] E. M. Thaning, M. L. M. Asplund, T. A. Nyberg, O. W. Inganas, H. von Hoist, J. Biomed. Mater. Res. B 2010, 93B, 407.

[59] T. Hadlock, C. Sundback, D. Hunter, M. Cheney, J. P. Vacanti, Tissue Eng. 2000, 6, 119

[60] V. Mukhatyar, L. Karumbaiah, J. Yeh, R. Bellamkonda, Adv. Mater. 2009, 21, 4670.

[61] J. M. Corey, D. Y. Lin, K. B. Mycek, Q. Chen, S. Samuel, E. L. Feldman, D. C. Martin, J. Biomed. Mater. Res. A 2007, 83A, 636

[62] S. Yoshii, M. Oka, M. Shima, A. Taniguchi, M. Akagi, Brain Res. 2002, 949, 202.

[63] X. Cao, M. S. Shoichet, Neuroscience 2001, 103, 831.

[64] S. Y. Chew, R. F. Mi, A. Hoke, K. W. Leong, Adv. Funct. Mater. 2007 $17,1288$.

[65] T. M. Brushart, P. N. Hoffman, R. M. Royall, B. B. Murinson, C. Witzel, T. Gordon, J. Neurosci. 2002, 22, 6631.

[66] E. G. Fine, R. F. Valentini, R. Bellamkonda, P. Aebischer, Biomaterials 1991, 12, 775.

[67] A. Mosahebi, P. Fuller, M. Wiberg, G. Terenghi, Exp. Neurol. 2002, $173,213$.

[68] X. Nie, Y. J. Zhang, W. D. Tian, M. Jiang, R. Dong, J. W. Chen, Y. Jin, Int. J. Oral. Maxillofac. Surg. 2007, 36, 32.

[69] N. Gomez, J. Y. Lee, J. D. Nickels, C. E. Schmidt, Adv. Funct. Mater 2007, 17, 1645

[70] J. Y. Lee, C. A. Bashur, A. S. Goldstein, C. E. Schmidt, Biomaterials 2009, 30, 4325.

[71] T. J. Rivers, T. W. Hudson, C. E. Schmidt, Adv. Funct. Mater. 2002 12, 33. 